\title{
Harmonic-Mean-Based Dual-Antenna Selection with Distributed Concatenated Alamouti Codes in Two-Way Relaying Networks
}

\author{
Guo Li ${ }^{1}$, Feng-Kui Gong ${ }^{1 *}$, Xiang Chen ${ }^{1}$ \\ ${ }^{1}$ State Key Laboratory of ISN, Xidian University \\ Xi'an, Shaanxi 710071 - China \\ [e-mail: liguoxidian@163.com, fkgong@xidian.edu.cn, chenx@stu.xidian.edu.cn] \\ *Corresponding author: Feng-Kui Gong
}

Received September 14, 2018; revised September 30, 2018; accepted November 3, 2018; published April 30, 2019

\begin{abstract}
In this letter, a harmonic-mean-based dual-antenna selection scheme at relay node is proposed in two-way relaying networks (TWRNs). With well-designed distributed orthogonal concatenated Alamouti space-time block code (STBC), a dual-antenna selection problem based on the instantaneous achievable sum-rate criterion is formulated. We propose a low-complexity selection algorithm based on the harmonic-mean criterion with linearly complexity $O\left(N_{R}\right)$ rather than the directly exhaustive search with complexity $O\left(N_{R}^{2}\right)$. From the analysis of network outage performance, we show that the asymptotic diversity gain function of the proposed scheme achieves as $1 / \rho^{N_{R}-1}$, which demonstrates one degree loss of diversity order compared with the full diversity. This slight performance gap is mainly caused by sacrificing some dual-antenna selection freedom to reduce the algorithm complexity. In addition, our proposed scheme can obtain an extra coding gain because of the combination of the well-designed orthogonal concatenated Alamouti STBC and the corresponding dual-antenna selection algorithm. Compared with the common-used selection algorithms in the state of the art, the proposed scheme can achieve the best performance, which is validated by numerical simulations.
\end{abstract}

Keywords: Harmonic-mean, dual-antenna selection, distributed concatenated Alamouti codes, two-way relaying networks, diversity gain function

This research was supported by Joint Fund of Ministry of Education of the People's Republic of China (6141A02022338), the National High-tech R\&D Program of China, Grant No. 2014AA01A704, the National Natural Science Foundation of China, Grant No. 61372067. 


\section{Introduction}

Wireless relaying systems have attracted much attention for the current and the future communication networks [1][2]. For a higher spectrum efficiency, two-way relaying networks (TWRNs) were proposed in [3][4], which just need two transmission phases to complete the bidirectional signal transmission and reception. Specifically, this is accomplished by simultaneously transmitting from the sources to the relay in the multi-access phase and by broadcasting the processed information from the relay to the sources in the broadcast phase. To achieve high diversity gain and coding gain, distributed space-time block codes (DSTBCs) are extensively studied in [5][6] where the relaying networks consist of multiple nodes or multiple antennas. These DSTBCs are generally designed to obtain the best performance improvement by constructing the distinctive space-time code structure[7][8][9].

Antenna selection scheme is an effective method to reduce the system resources while keeping a moderate performance [10]. Most of articles can be categorized into two groups. The one is to peruse the optimal antenna selection algorithm without performance loss. Single antenna selection scheme has been generally studied, and the relative analyses have shown that the same diversity gain can be achieved as the all-antenna-used system. The other focuses on reducing the realization complexity. The optimal antenna selection algorithm is exhaustive searching according to some performance metric among all the candidate antenna set. However, its complexity is generally prohibitive, especially in large number of antennas or in the relaying networks.

As we know, the space-time code transmission strategies not only have remarkable diversity gain, but also bring the coding gain. In this letter, we expect to obtain the diversity gain by using low-complexity antenna selection scheme, while to achieve the coding gain by using some space-time code transmission strategy. Therefore, multi-antenna selection combined with space-time code transmission is naturally studied.

In this letter, we concentrate on dual-antenna selection combining with DSTC at the relay node. Based on our well-designed distributed concatenated Alamouti space-time block code (STBC), we aim to study the dual-antenna selection algorithm based on instantaneous achievable sum-rate of overall network. Meanwhile, considering the implementation complexity, we also propose a low-complexity selection strategy, which may need to make some tradeoff between the algorithm complexity and the network performance.

The rest of this paper is organized as follows. The related works are presented in Section 2. The system model and the optimal antenna selection criterion are shown in Section 3. In Section 4, the near-optimal harmonic-mean-based dual-antenna selection scheme is proposed. Performance analysis and numerical simulation results are shown in Section 5 and Section 6, respectively. Finally, the conclusions are drawn in Section 7. 


\section{Related Work}

Although many benefits of multi-antenna systems have been verified, the deployment of multiple antennas requires multiple radio frequency (RF) chains. These RF chains include multiple analog-digital converters, low noise amplifiers, down-converters, etc., whose high cost is undesirable especially for mobile handsets. To reduce the number of RF-chains and keep the system simple and inexpensive, several antenna selection (AS) algorithms are proposed to feed the most favorable transmit and/or receive antennas [10-17]. In two-way networks, AS has also been extensively considered. In [12], single antenna selection scheme at source node was studied and compared with the beamforming, which shows that the same diversity order can be obtained for both schemes. In [13][14], joint relay and antenna selection was discussed over Nakagamin fading channels. Greedy-based AS scheme is presented in [15] and the theoretical analysis about joint relay and antenna selection is shown in [16]. A max-min-based approach for relay AS was proposed in [17], which selected single antenna at the relay node to maximise the minimum end-to-end receiving signalto-noise ratio (SNR). A minimum mean square error based greedy AS algorithm was proposed for amplify-and-forward (AF) MIMO relaying systems [18], which adopted an iterative selection algorithm to minimise the mean square error. Recently, AS combined with interference alignment (IA) scheme [19] was newly studied, which greatly improves the received SINR of each user in cognitive radio networks. Sum-rate maximization scheme by using the second-order cone programming was studied in [20], which showed a promising algorithm to guarantee the secure transmission of primary user when the spectrum is shared with secondary users.

However, most AS schemes in the state of the art generally consider selecting single antenna at the source node or relay node. There are no reports on combining the multi-antenna selection and the DSTBCs in relaying networks.

\section{Distributed Concatenated Alamouti Codes with Dual-Antenna Selection at Relay Node}

In this paper, we consider a two-way relaying network in which the source nodes $T_{1}$ and $T_{2}$, communicate with the help of an intermediate relaying node $R$ by using the amplify-and-forward (AF) protocol. Each source node is equipped with single antenna, while the relay $R$ is equipped with $N_{R}$ antennas. We assume the channels between the sources and the relay satisfy Rayleigh fading distribution, and there is no direct link between $T_{1}$ and $T_{2}$, in case the sources are located far away from each other or within the deep fading areas. We denote the channel fading vector between $T_{1}$ and $R$ as $\mathbf{h}=\left[h_{1}, h_{2}, \cdots, h_{N_{R}}\right]^{T}$ and the channel fading vector between $T_{2}$ and $R$ as $\mathbf{g}=\left[g_{1}, g_{2}, \cdots, g_{N_{R}}\right]^{T}$, where each channel fading element is modeled as independent identically distributed Rayleigh fading $\mathcal{C N}(0,1)$. 


\subsection{System Model}

As shown in Fig. 1, in our two-way relaying network, two antennas are selected at the relay node $R$ according to carefully designed selection criterion. There are mainly two transmission phases in one whole information exchange between source nodes $T_{1}$ and $T_{2}$, i.e., multiple accessing (MA) phase at the two sources and broadcasting (BC) phase at the relay node.

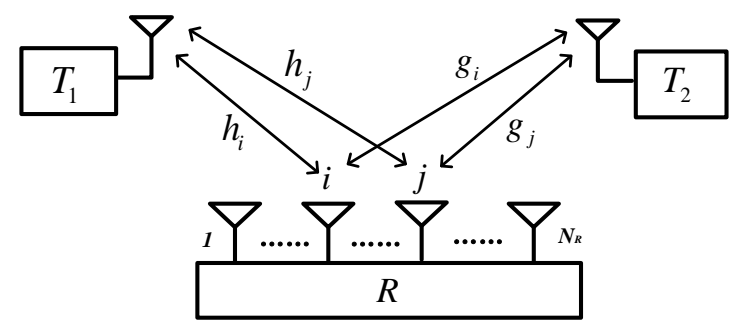

Fig. 1. Two-way relaying network with dual-antenna selection

The source $T_{1}$ transmits $\mathbf{s}_{1}=\left[s_{11}, s_{12}^{*}\right]^{T}$ and the source $T_{2}$ transmits $\mathbf{s}_{2}=\left[s_{21}, s_{22}^{*}\right]^{T}$ to relay $R$ simultaneously in consecutive two time slots. Here, we assume the $i$-th and $j$-th antennas at $R$ are selected to receive signals. Therefore, the received signals at $R$ via the selected antennas can be expressed as

$$
\begin{aligned}
& \mathbf{r}_{1}=\mathbf{h}_{s e l}^{(i, j)} s_{11}+\mathbf{g}_{s e l}^{(i, j)} s_{21}+\mathbf{n}_{R 1}, \\
& \mathbf{r}_{2}=\mathbf{h}_{s e l}^{(i, j)} s_{12}^{*}+\mathbf{g}_{s e l}^{(i, j)} s_{22}^{*}+\mathbf{n}_{R 2},
\end{aligned}
$$

where $\mathbf{h}_{\text {sel }}^{(i, j)}=\left[h_{i}, h_{j}\right]^{T}$ and $\mathbf{g}_{s e l}^{(i, j)}=\left[g_{i}, g_{j}\right]^{T}$ denote the selected channel vectors between $T_{1}, T_{2}$ and $R$ respectively, $\mathbf{n}_{R 1}$ and $\mathbf{n}_{R 2}$ denote the noise vectors at $R$ with each element having zero mean and variance $\sigma^{2}$.

\section{B. Broadcasting (BC) Phase}

The relay $R$ first processes the received signals by using a linear combination matrix $\mathbf{A}$ as follows

$$
\mathbf{t}=\mathbf{r}_{1}+\mathbf{A r}_{2}^{*} \text {, }
$$

where $\mathbf{t}=\left[t_{1}, t_{2}\right]^{T}, \mathbf{A}=[0-1 ; 10]$. Then, $R$ broadcasts $\mathbf{t}$ by using Alamouti code in consecutive two time slots via the selected antennas. Specially, in the first time slot $R$ broadcasts $t_{1}$ and $t_{2}$ from the $i$-th and $j$-th antennas respectively, and in the second time slot $R$ broadcasts $t_{2}^{*}$ and $-t_{1}^{*}$. Thus, for the source node $T_{1}$, the received signals can be obtained as

$$
\begin{aligned}
& y_{1}=\beta h_{i} t_{1}+\beta h_{j} t_{2}+n_{11}, \\
& y_{2}=\beta h_{i} t_{2}^{*}-\beta h_{j} t_{1}^{*}+n_{12},
\end{aligned}
$$

where $\beta$ denotes the power scaling factor at $R$, i.e.,

$$
\beta=\frac{1}{\sqrt{2}}\left(\left\|\mathbf{h}_{\text {sel }}^{(i, j)}\right\|^{2}+\left\|\mathbf{g}_{\text {sel }}^{(i, j)}\right\|^{2}+2 \sigma^{2}\right)^{-1 / 2} .
$$


The received signals at source node $T_{2}$ can be obtained similarly. Combining (1)-(5) and removing the self-interference, the received signals at $T_{1}$ and $T_{2}$ can be expressed as

$$
\begin{array}{ll}
T_{1}: & \mathbf{y}_{1}=\beta \mathbf{H}^{T} \mathbf{G} \mathbf{s}_{2}+\xi_{1}, \\
T_{2}: & \mathbf{y}_{2}=\beta \mathbf{G}^{T} \mathbf{H} \mathbf{s}_{1}+\xi_{2},
\end{array}
$$

where the equivalent channel matrices $\mathbf{H}$ and $\mathbf{G}$ can be expressed as

$$
\mathbf{H}=\left[\begin{array}{cc}
h_{i} & -h_{j}^{*} \\
h_{j} & h_{i}^{*}
\end{array}\right], \quad \mathbf{G}=\left[\begin{array}{cc}
g_{i} & -g_{j}^{*} \\
g_{j} & g_{i}^{*}
\end{array}\right],
$$

the equivalent noise vectors $\xi_{1}$ and $\xi_{2}$ are complex Gaussian random vectors with distribution

$$
\boldsymbol{\xi}_{1} \sim \mathcal{C N}\left(\mathbf{0},\left(2 \beta^{2} \sigma^{2}\left(\left|h_{i}\right|^{2}+\left|h_{j}\right|^{2}\right)+1\right) \mathbf{I}_{2}\right)
$$

and

$$
\boldsymbol{\xi}_{2} \sim \mathcal{C N}\left(\mathbf{0},\left(2 \beta^{2} \sigma^{2}\left(\left|g_{i}\right|^{2}+\left|g_{j}\right|^{2}\right)+1\right) \mathbf{I}_{2}\right) .
$$

In addition, $\mathbf{H}^{T} \mathbf{G}$ and $\mathbf{G}^{T} \mathbf{H}$ are all orthogonal Alamouti matrices which will greatly simplify the maximum-likelihood (ML) detection to symbol-by-symbol detection. We call this space-time code as distributed concatenated Alamouti codes. Substituting $\beta$ into (6) and (7), the instantaneous end-to-end received SNR at $T_{1}$ and $T_{2}$ can be written as

$$
\begin{aligned}
& \gamma_{1}=\frac{\left(\left|h_{i}\right|^{2}+\left|h_{j}\right|^{2}\right)\left(\left|g_{i}\right|^{2}+\left|g_{j}\right|^{2}\right)}{2\left(2\left|h_{i}\right|^{2}+2\left|h_{j}\right|^{2}+\left|g_{i}\right|^{2}+\left|g_{j}\right|^{2}\right) \sigma^{2}}, \\
& \gamma_{2}=\frac{\left(\left|h_{i}\right|^{2}+\left|h_{j}\right|^{2}\right)\left(\left|g_{i}\right|^{2}+\left|g_{j}\right|^{2}\right)}{2\left(\left|h_{i}\right|^{2}+\left|h_{j}\right|^{2}+2\left|g_{i}\right|^{2}+2\left|g_{j}\right|^{2}\right) \sigma^{2}} .
\end{aligned}
$$

We note that $\gamma_{1}$ and $\gamma_{2}$ are not statistically independent with each other, which are all related to the selected antennas.

\subsection{Dual-Antenna Selection Criterion at Relay Node}

To effectively evaluate the network performance, we define the instantaneous achievable sum-rate of overall network as

$$
R_{\text {sum }}=\frac{1}{2} \log _{2}\left(1+\gamma_{1}\right)+\frac{1}{2} \log _{2}\left(1+\gamma_{2}\right)
$$

Therefore, the optimal dual-antenna selection criterion is to maximize the instantaneous achievable rate $\mathcal{R}$, i.e.,

$$
\left(i^{*}, j^{*}\right)=\arg \max _{i, j \in\left\{1,2, \cdots N_{R}\right\}, i \neq j} R_{\text {sum }} .
$$

Remarks: the two optimal antennas could be selected by exhaustive search with a computational complexity $O\left(N_{R}^{2}\right)$, which is not efficient especially when $N_{R}$ is large. 
Therefore, we expect to propose a low-complexity and pragmatic dual-antenna selection algorithm in the next section.

\section{Harmonic-Mean-Based Dual-Antenna Selection Algorithm}

By using the inequality of arithmetic and geometric means ${ }^{1}$, the sum rate (10) can be bounded as

$$
R_{\text {sum }}=\frac{1}{2} \log _{2}\left(1+\gamma_{1}+\gamma_{2}+\gamma_{1} \gamma_{2}\right) \geq \log _{2}\left(1+\sqrt{\gamma_{1} \gamma_{2}}\right) .
$$

Combining with (8) and (9), and further using the inequality $1 / a+1 / b \geq 4 /(a+b), a \geq 0, b \geq 0$, we have

$$
\begin{aligned}
\mathcal{R} & \geq \log _{2}\left(1+\frac{1}{\sigma^{2}}\left(\frac{1}{2\left|h_{i}\right|^{2}}+\frac{1}{2\left|h_{j}\right|^{2}}+\frac{1}{\left|g_{i}\right|^{2}}+\frac{1}{\left|g_{j}\right|^{2}}\right)^{-\frac{1}{2}}\left(\frac{1}{\left|h_{i}\right|^{2}}+\frac{1}{\left|h_{j}\right|^{2}}+\frac{1}{2\left|g_{i}\right|^{2}}+\frac{1}{2\left|g_{j}\right|^{2}}\right)^{-\frac{1}{2}}\right) \\
& \geq \log _{2}\left(1+\frac{4}{3 \sigma^{2}}\left(\frac{1}{\left|h_{i}\right|^{2}}+\frac{1}{\left|g_{i}\right|^{2}}+\frac{1}{\left|h_{j}\right|^{2}}+\frac{1}{\left|g_{j}\right|^{2}}\right)^{-1}\right) .
\end{aligned}
$$

For more tractable analysis, we alternatively consider the dual-antenna selection criterion as maximizing this lower bound of instantaneous achievable sum rate. Consequently, we propose a near-optimal harmonic-mean-based dual-antenna selection algorithm as the following Lemma.

Lemma 1: A near-optimal harmonic-mean-based dual-antenna selection algorithm based on (11) is expressed as

$$
\begin{aligned}
& i^{*}=\underset{i \in\left\{1,2, \cdots N_{R}\right\}}{\operatorname{argmax}} \mu_{H}\left(\left|h_{i}\right|^{2},\left|g_{i}\right|^{2}\right), \\
& j^{*}=\underset{j \in\left\{\left\{, 2, \cdots N_{R}\right\}, j \neq i^{*}\right.}{\operatorname{argmax}} \mu_{H}\left(\left|h_{j}\right|^{2},\left|g_{j}\right|^{2}\right),
\end{aligned}
$$

where $\mu_{H}(X, Y)=2 X Y /(X+Y)$ is the harmonic mean of $X>0, Y>0$.

Compared with (11), Lemma 1 alternatively converts the original two-dimension optimization problem to a one-dimension search, which achieves a linear selection complexity $O\left(N_{R}\right)$. Fig. 2 illustrates the comparisons of selection complexity ${ }^{2}$ between two algorithms. We can see the harmonic-mean-based dual-antenna selection is more efficient especially for large $N_{R}$.

\footnotetext{
1 The inequality of arithmetic and geometric means: $a+b \geq 2 \sqrt{a b}, a \geq 0, b \geq 0$.

${ }^{2}$ Here, we mainly consider the average times of computations for the two optimal selected antennas.
} 


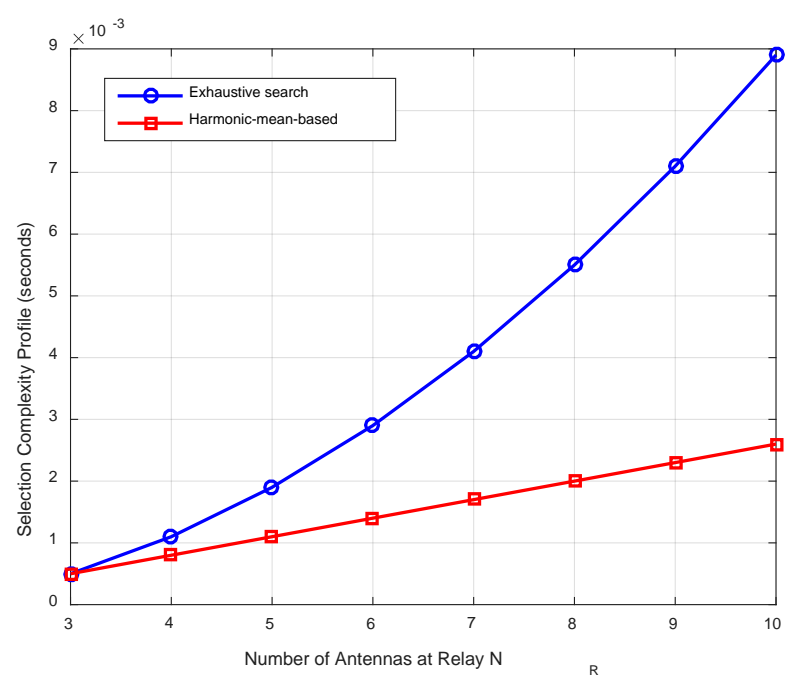

Fig. 2. Complexity comparisons between the proposed harmonic-mean-based AS and the exhaustive search AS

\section{Performance Analysis}

In this section, a sum-rate outage probability upper bound is derived based on the proposed dual-antenna selection algorithm in Lemma 1 . We define the sum-rate outage happens only when sum-rate $R_{\text {sum }}$ is below a given threshold $R_{\text {sum }}^{\text {th }}$. For analytical tractability, we consider the lower bounded sum-rate presented in (14) which consequently results in an outage probability upper bound as follows.

$$
P_{\text {out }}^{U}\left(R_{\text {sum }}^{\text {th }}\right)=P\left\{\frac{1}{\left|h_{i^{*}}\right|^{2}}+\frac{1}{\left|g_{i^{*}}\right|^{2}}+\frac{1}{\left|h_{j^{*}}\right|^{2}}+\frac{1}{\left|g_{j^{*}}\right|^{2}} \geq \frac{4}{3\left(2^{R_{\text {sum }}}-1\right) \sigma^{2}}\right\} .
$$

Before further analysis and discussions, we first provide the following lemma.

Lemma 2: Let $Z_{1}=1 /\left|h_{i^{*}}\right|^{2}+1 /\left|g_{i^{*}}\right|^{2}, Z_{2}=1 /\left|h_{j^{*}}\right|^{2}+1 /\left|g_{j^{*}}\right|^{2}$, the joint PDF is written as

$$
f_{z_{1} z_{2}}\left(z_{1}, z_{1}\right)=\frac{4 N_{R}\left(N_{R}-1\right)}{z_{1}^{2} z_{2}^{2}}\left[1-\frac{2}{z_{2}} K_{1}\left(\frac{2}{z_{2}}\right) e^{-\frac{2}{z_{2}}}\right]^{N_{R}-2} e^{-\frac{2}{z_{1}}-\frac{2}{z_{2}}}
$$

where $K_{v}(s)$ denotes the first order modified bessel function of the second kind [21].

Proof: The proof is presented in Appendix A.

Let $z_{t h}=4 / 3\left(2^{R_{s u m}^{\text {th }}}-1\right) \sigma^{2}$, then (17) can be expressed as

$$
\begin{aligned}
P_{\text {out }}^{U}\left(R_{\text {sum }}^{\text {th }}\right) & =P\left\{z_{1}+z_{2} \geq z_{\text {th }} \mid z_{1} \leq z_{2}\right\} \\
& =\underbrace{\int_{z_{1}=0}^{\frac{z_{\text {th }}}{2}} \int_{z_{2}=z_{\text {th }}-z_{1}}^{\infty} f_{z_{1} z_{2}}\left(z_{1}, z_{2}\right) d z_{1} d z_{2}}_{A}+\underbrace{\int_{z_{1}}^{\infty} \frac{z_{\text {th }}}{2} \int_{z_{2}=z_{1}}^{\infty} f_{z_{1} z_{2}}\left(z_{1}, z_{2}\right) d z_{1} d z_{2}}_{B}
\end{aligned}
$$


Using Lemma 1 and the approximations $K_{v}(s) \sim \Gamma(s) / 2(s / 2)^{-v}$ and $1-e^{-s} \approx s$ for small $s$, we have

$$
\begin{aligned}
A= & 2^{N_{R}} N_{R}\left(N_{R}-1\right) \int_{z_{1}=0}^{\frac{z_{\text {th }}}{2}} \int_{z_{\text {th }}-z_{1}}^{\infty} \frac{1}{z_{1}^{2} z_{2}^{N_{R}}} e^{-\frac{2}{z_{1}}-\frac{2}{z_{2}}} d z_{2} d z_{1} \\
& =2 N_{R}\left(N_{R}-1\right) \int_{z_{1}=0}^{\frac{z_{\text {th }}}{2}} \frac{e^{-2 z_{1}^{-1}}}{z_{1}^{2}} \gamma\left(N_{R}-1, \frac{2}{z_{\text {th }}-z_{1}}\right) d z_{1},
\end{aligned}
$$

where $\gamma(n, x)$ denotes the incomplete gamma function which can be approximated as

$$
\gamma(n, x)=x^{n} e^{-x} \sum_{k=0}^{\infty} x^{k} /(n(n+1) \cdots(n+k)) \approx x^{n} e^{-x} / n,
$$

where we just take the first summand in the above infinite series when $x$ is large. Thus, we obtain

$$
\begin{aligned}
A= & 2^{N_{R}} N_{R} Z_{t h}^{-\left(N_{R}-1\right)} \int_{t=\frac{2}{z_{t h}}}^{\infty}\left(\frac{z_{t h} t}{z_{t h} t-1}\right)^{N_{R}-1} e^{-2 t} d t \\
& \approx 2^{N_{R}-1} N_{R} e^{-\frac{4}{z_{t h}}} z_{t h}^{-\left(N_{R}-1\right)}+O\left(z_{t h}^{-N_{R}}\right) .
\end{aligned}
$$

Similarly, we have

$$
B=2^{2 N_{R}} e^{-\frac{8}{z_{t h}}} z_{t h}^{-N_{R}}+O\left(z_{t h}^{-\left(N_{R}+1\right)}\right) .
$$

Consequently, we have the sum-rate outage probability upper bound as the following theorem.

Theorem 1: The upper bound of sum-rate outage probability based on our proposed harmonic-mean dual-antenna selection can be obtained as

$$
P_{\text {out }}\left(R_{\text {sum }}^{\text {th }}\right) \leq C \rho^{-\left(N_{R}-1\right)}+O\left(\rho^{-N_{R}}\right)
$$

where $C=N_{R}(3 / 2)^{N_{R}-1}\left(2^{2^{\text {psum }}}-1\right)^{N_{R}-1}, \rho=1 / \sigma^{2}$.

From Theorem 1, we can clearly see the proposed harmonic-mean dual-antenna selection can achieves the diversity gain function $1 / \rho^{N_{R}-1}$ at least. Compared with full diversity performance with the same network deployment, which claimed a lower bound of diversity gain function being $\log _{e} \rho / \rho^{N_{R}}$, one degree loss of diversity gain in proposed scheme is mainly ascribed to some sacrifice of selection freedom for a low-complexity algorithm.

\section{Numerical Results}

In this section, we provide simulations to evaluate the performance of the proposed harmonic-mean-based dual-antenna selection scheme, and to validate the theoretical analysis of the diversity gain function of the outage probability. We consider the independent identical distributed Rayleigh fading channels as described in Section 2. 
Firstly, we carry out the sum-rate simulations. As shown in Fig. 3 and Fig. 4, compared with the common-used antenna selection algorithms, such as max-min antenna selection [12][13], geometric antenna selection, arithmetic antenna selection, our proposed harmonic-mean-based dual-antenna selection scheme can obtain the best sum-rate. Especially, from the simulation results in Fig. 4, we observe that the sum-rate improvement is remarkable when the number of antennas is large.

Secondly, Fig. 5 shows the comparisons of outage probability performance between the proposed scheme and the other antenna selection schemes. From the results, our proposed harmonic-mean-based dual-antenna selection scheme outperforms the max-min antenna selection used in [12][13]. In addition, with the similar procedures, the arithmetic-mean-based and geometric-mean-based selection schemes are also simulated, which show the harmonic-mean-based selection scheme achieves apparent superiority. Fig. 6 shows the outage probability performance with increasing number of antennas, which also validates the improved performance of our proposed scheme.

Finally, Fig. 7 shows the upper outage probability performance for different antenna configurations. The simulated results based on equation (17) for $N_{R}=4,6,8$ achieve perfect agreement with the theoretical analysis when SNR is large, which directly verify the correctness of Theorem 1.

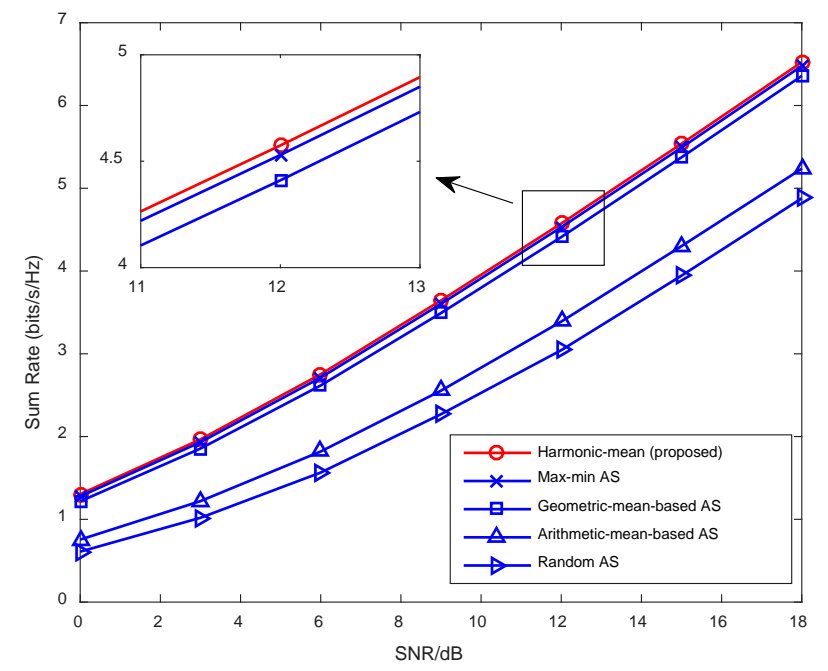

Fig. 3. Numerical simulations of sum-rate with the increasing SNR, compared with common-used antenna selection algorithms, $N_{R}=10$. 


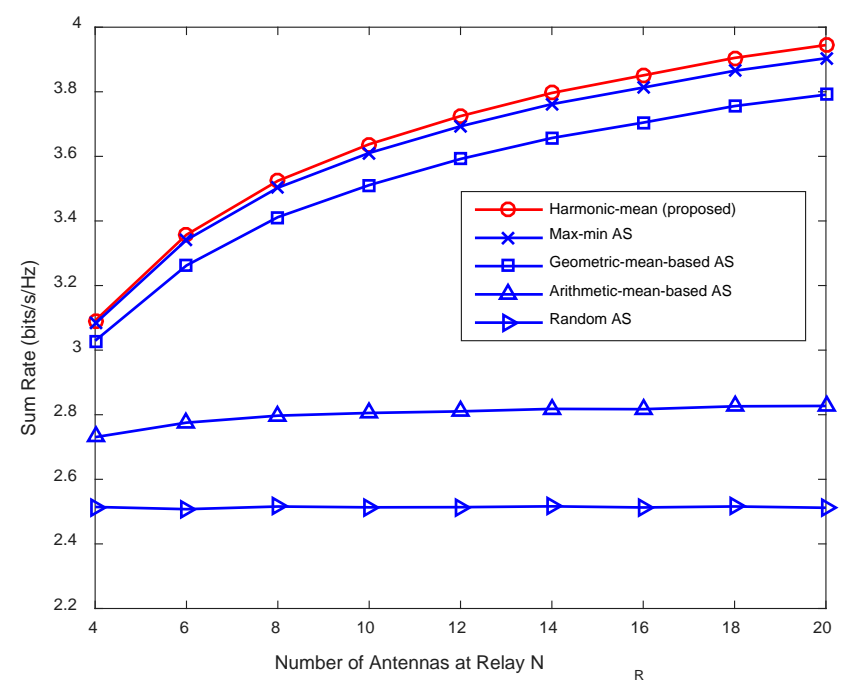

Fig. 4. Numerical simulations of throughputs with the increasing number of antennas, compared with common-used antenna selection algorithms, $S N R=10 \mathrm{~dB}$.

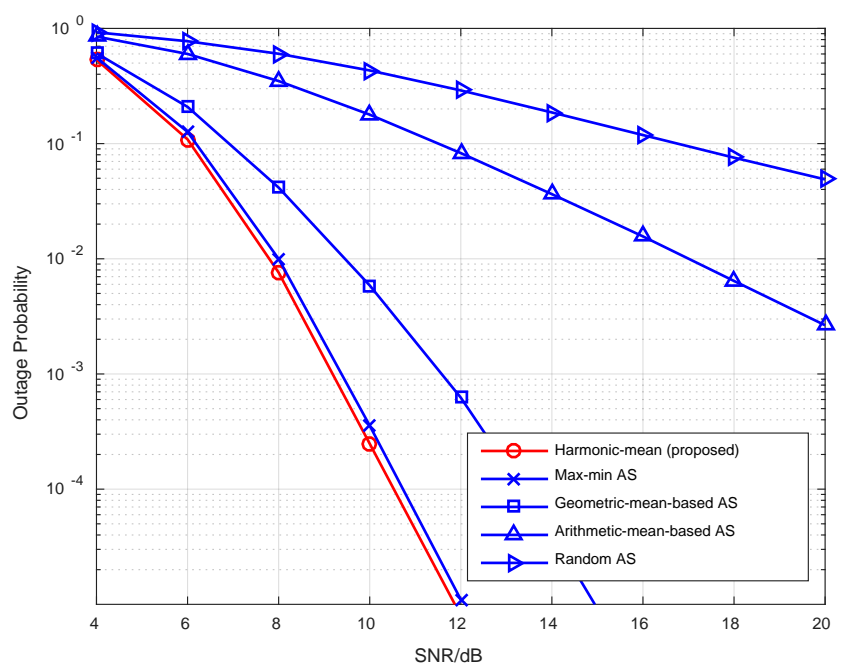

Fig. 5. Outage performance with the increasing SNR, compared with common-used antenna selection algorithms, $N_{R}=10, R_{\text {sum }}^{\text {th }}=2$. 


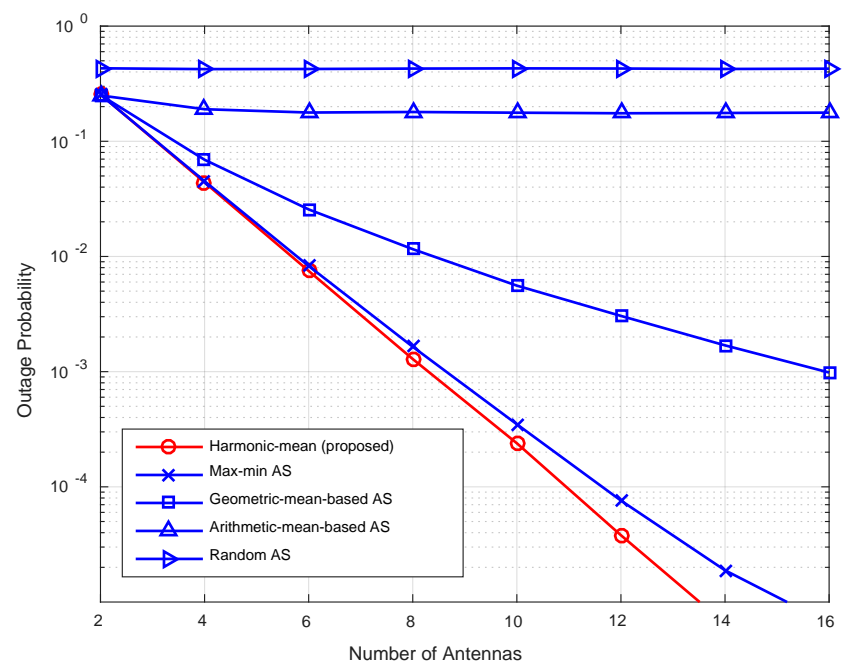

Fig. 6. Outage performance with the increasing number of antennas, compared with common-used antenna selection algorithms, $S N R=10 \mathrm{~dB}, R_{\text {sum }}^{\text {th }}=2$.

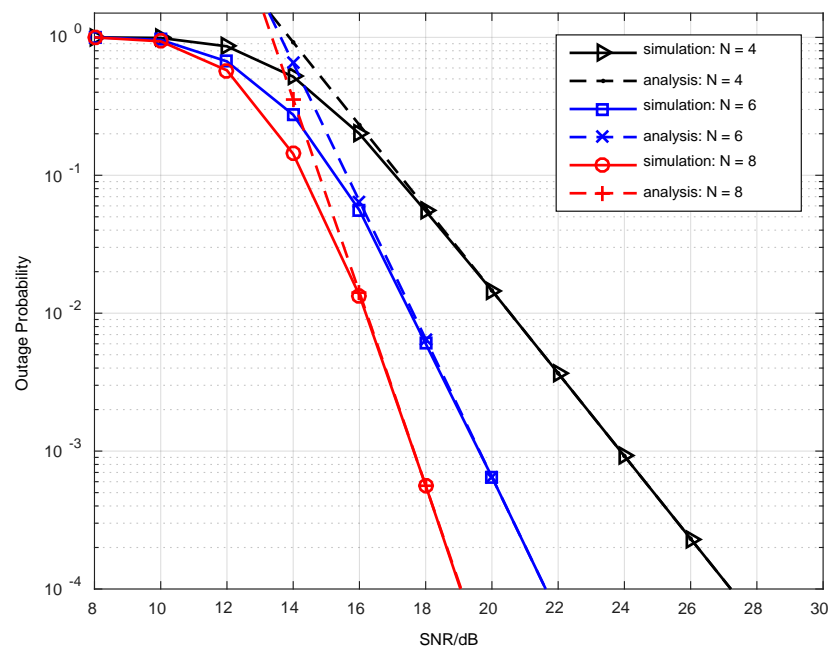

Fig. 7. Outage probability performance of harmonic-mean dual-antenna selection, simulation results vs. theory analyses, $N_{R}=4,6,8$ with $R_{\text {sum }}^{\text {th }}=3$

\section{Conclusion}

In this paper, we have proposed a harmonic-mean-based dual-antenna selection scheme for the two-way relaying networks. Combining with the well-designed distributed orthogonal concatenated Alamouti codes, we alternatively convert the optimal dual-antenna selection to a near-optimal linear-complexity selection algorithm. From the asymptotic analysis, we demonstrate the proposed scheme achieves the diversity gain function $1 / \rho^{N_{R}-1}$ at least. Numerical results verify our analysis and provide insights into the outperformed performance of the proposed scheme. 


\section{Appendix}

A. Proof of Lemma 2

From Lemma 1, we can find

$$
\arg \max _{i} \mu_{H}\left(\left|h_{i}\right|^{2},\left|g_{i}\right|^{2}\right)=\arg \min _{i}\left(\frac{1}{\left|h_{i}\right|^{2}}+\frac{1}{\left|g_{i}\right|^{2}}\right) .
$$

Defining $U=\left|h_{i}\right|^{2}, V=\left|g_{i}\right|^{2}, X=1 /\left|h_{i}\right|^{2}, Y=1 /\left|g_{i}\right|^{2}$, we have their PDFs as

$$
f_{U}=e^{-u}, \quad f_{V}=e^{-v}, \quad f_{X}=\frac{1}{x^{2}} e^{-\frac{1}{x}}, \quad f_{Y}=\frac{1}{y^{2}} e^{-\frac{1}{y}} .
$$

Let $Z=X+Y$, its CDF can be calculated as

$$
F_{Z}(z)=\int_{x=0}^{z} \int_{y=0}^{z-x} \frac{1}{x^{2} y^{2}} e^{-\frac{1}{x}} e^{-\frac{1}{y}} d x d y=\int_{x=0}^{z} \frac{1}{x^{2}} e^{-\frac{z}{x(z-x)}} d x=\frac{2}{z} e^{-\frac{2}{z}} K_{1}\left(\frac{2}{z}\right)
$$

where the second equation above comes from reference [21, 3.324.1]. Consequently, the PDF of $Z$ can be obtained as $f_{Z}(z)=2 z^{-2} e^{-2 / z}$, where we simplify the expression with approximation $K_{v}(z) \sim 1 / 2 \Gamma(v)(z / 2)^{-v}$. Combining Lemma 1, we can find that the selected $i^{*}$ and $j^{*}$ based on harmonic-mean yield the minimum and the second smallest values of random variable $Z$ respectively, i.e. $Z_{1}, Z_{2}$. Therefore, with the theory of Order Statistics [22], the joint PDF of $Z_{1}$ and $Z_{2}$ can be expressed as

$$
f_{z_{1} z_{2}}\left(z_{1}, z_{2}\right)=N(N-1)\left[1-F\left(z_{2}\right)\right]^{N-2} f\left(z_{1}\right) f\left(z_{2}\right)
$$

This Complete the proof of Lemma 2.

\section{References}

[1] F. Boccardi, R. W. Heath, A. Lozano, T. L. Marzetta and P. Popovski, "Five disruptive technology directions for 5G,” IEEE Communications Magazine, vol. 52, no. 2, pp. 74-80, February 2014. Article (CrossRef Link).

[2] A. Gupta and R. Jha, "A survey of 5G network: architecture and emerging technologies,” IEEE Access, vol. 3, pp. 1206-1232, July 2015. Article (CrossRef Link).

[3] B. Rankov and A. Wittneben, "Spectral efficient protocol for half-duplex fading relay channels," IEEE Journal on Selected Areas in Communications, vol. 25, no.2, pp. 379-389, February 2007. Article (CrossRef Link).

[4] Y. Han ; S. H. Ting; C. K. Ho and W. H. Chin, "Performance bounds for two-way amplify-and-forward relaying,” IEEE Transactions on Wireless Communications, vol. 8, no. 1, pp. 432-439, February 2009. Article (CrossRef Link).

[5] Y. Jing and B. Hassibi, "Distributed space-time coding in wireless relay networks," IEEE Transactions on Wireless Communications, vol. 5, no.12, pp. 3524-3536, December 2006. Article (CrossRef Link).

[6] F. Gong, J. Zhang and J. Ge, "Novel distributed quasi-orthogonal space-time block codes for two-way two-antenna relay networks," IEEE Transactions on Wireless Communications, vol. 12, no.9, pp. 4338-4349, August 2013. Article (CrossRef Link). 
[7] K. Unnikrishnan and B. Rajan, "Space-time coded spatial modulated physical layer network coding for two-way relaying," IEEE Transactions on Wireless Communications, vol. 14, no. 1, pp. 331-342, August 2015. Article (CrossRef Link).

[8] H., Yan and H. Nguyen, "Distributed space time coding for bit-interleaved coded modulation in two-way relaying communications,” in Proc. of IEEE Globecom, pp. 1-6, December 2015. Article (CrossRef Link).

[9] H. M. Wang, Q. Yin and X.-G. Xia, "Distributed beamforming for physical-layer security of two-way relay networks,” IEEE Transactions on Signal Processing, vol. 60, no.7, pp. 3532-3545, July 2012. Article (CrossRef Link).

[10] I. Bahceci, T. M. Duman and Y. Altunbasak, “Antenna selection for multiple-antenna transmission systems: performance analysis and code construction,” IEEE Transactions on Information Theory, vol. 49, no.10, pp. 2669-2681, October 2003. Article (CrossRef Link).

[11] N. Yang; P. L. Yeoh, M. Elkashlan, R. Schober and I. B. Collings, "Transmit antenna selection for security enhancement in MIMO wiretap channels,” IEEE Transactions on Communications, vol. 61, no. 1, pp. 144-154, October 2013. Article (CrossRef Link).

[12] N. Yang, P. L. Yeoh, M. Elkashlan, I. B. Collings and Z. Chen, "Two-way relaying with multi-antenna sources: beamforming and antenna selection," IEEE Transactions on Vehicular Technology, vol. 61, no. 9, pp. 3996-4008, November 2012. Article (CrossRef Link).

[13] K. Yang, N. Yang, C. Xing and J. Wu, "Relay antenna selection in MIMO two-way relay networks over Nakagami-fading channels," IEEE Transactions on Vehicular Technology, vol. 63, no. 5, pp. 2349-2362, June 2014. Article (CrossRef Link).

[14] G. Amarasuriya, C. Tellambura, M. Ardakani, "Two-way amplify-andforward multiple-input multiple-output relay networks with antenna selection," IEEE Journal on Selected Areas in Communications, vol. 30, no. 8, pp. 1513-1529, September 2012. Article (CrossRef Link).

[15] X. Zhou, B. Bai and W. Chen, "Greedy relay antenna selection for sum rate maximization in amplify-and-forward MIMO two-way relay channels under a holistic power model,” IEEE Communications Letters, vol. 19, no. 9, pp. 1648-1651, September 2015. Article (CrossRef Link).

[16] K. Song, B. Ji, Y. Huang, M. Xiao and L. Yang, "Performance analysis of antenna selection in two-way relay networks,” IEEE Transactions on Signal Processing, vol. 63, no.10, pp. 2520-2532, May 2015. Article (CrossRef Link).

[17] M. Eslamifar, C. Yuen and W. Chin, "Max-min antenna selection for bidirectional multi-antenna relaying,” in Proc. of IEEE 71st VTC, pp. 1-5, May 16-19, 2010. Article (CrossRef Link).

[18] M. Ding, S. Liu and H. Luo, "MMSE based greedy antenna selection scheme for AF MIMO relay systems,” IEEE Signal Processing Letters, vol. 17, no. 5, pp. 433-436, May 2010. Article (CrossRef Link).

[19] X. Li, N. Zhao, Yi Sun and F. R. Yu, "Interference alignment based on antenna selection with imperfect channel state information in cognitive radio networks," IEEE Transactions on Vehicular Technology, vol. 65, no. 7, pp. 5497-5511, July 2016. Article (CrossRef Link).

[20] Y. Cao, N. Zhao, F. R. Yu, M. Jin, Y. Chen, J. Tang and V. C. M. Leung, “Optimization or alignment: secure primary transmission assisted by secondary networks,” Journal on selected areas in communications, vol. 36, no. 4, pp. 905-917, April 2018. Article (CrossRef Link).

[21] M. Abramowitz and I. A. Stegun, "Handbook of mathematical functions," Applied mathematics series, vol. 55, pp. 62, 1966.

[22] H. A. David and H. N. Nagaraja, “Order statistics,” Wiley Online Library, 1970. 


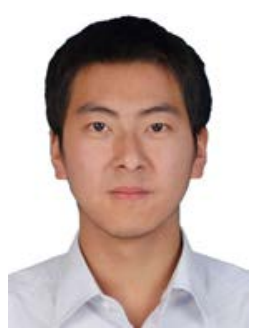

Guo Li was born in Shaanxi, China, in 1989. He received the B.Sc. and M.Sc. degrees in communications engineering from Xidian University, Xi'an, China, in 2011 and 2014, respectively. He is currently pursuing the Ph.D degree in communication and information system, Xidian University. His research interests include MIMO wireless communications, cooperative communications, and large-scale antenna system.

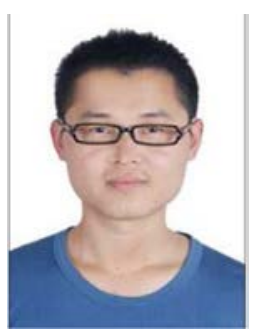

Feng-Kui Gong was born in Shandong, China, in 1979. He received the M.S. and Ph.D. degrees from Xidian University, Xi'an, China, in 2004 and 2007, respectively. From 2011 to 2012, he was a Visiting Scholar with the Department of Electrical and Computer Engineering, McMaster University, Hamilton, ON, Canada. He is currently a Professor with the Department of Communication Engineering, State Key Laboratory of Integrated Services Networks, Xidian University. His research interests include cooperative communication, distributed space-time coding, digital video broadcasting system, satellite communication, and 4G/5G techniques.

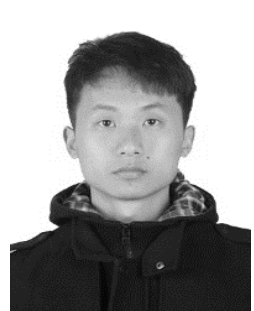

Xiang Chen was born in Hubei, China, in 1992. He received the B.Sc. degree in communications engineering from Dalian Maritime University, Dalian, China, in 2014, and M.Sc. degree in communications engineering from Xidian University, Xi'an, China, in 2014. He is currently pursuing the Ph.D degree in communication and information system, Xidian University. His research interests include Massive MIMO wireless communications, cooperative communications, and NOMA. 